\title{
Efecto del consumo de alcohol al diagnóstico en la sobrevida de pacientes con cirrosis hepática en un hospital universitario de la ciudad de Medellín
}

\section{Effect of alcohol consumption on diagnosis of liver cirrhosis patients' survival at a University Hospital in Medellín}

\author{
Diana Marcela Escobar, ${ }^{1}$ Juan Carlos Restrepo, ${ }^{1}$ Sergio Hoyos, ${ }^{1}$ María Cristina Navas, ${ }^{2}$
}

\author{
Grupo de Gastrohepatología, Facultad de Medicina, \\ Universidad de Antioquia. Hospital Pablo Tobón \\ Uribe, Medellín, Colombia. \\ 2 Hospital Pablo Tobón Uribe, Medellín, Colombia. \\ Correspondencia: Diana Marcela Escobar: diana. \\ escobar1@udea.edu.co \\ Fecha recibido: $28-04-17$ \\ Fecha aceptado: $07-05-18$
}

\begin{abstract}
Resumen
El consumo de alcohol es un conocido factor de riesgo para muerte prematura, morbilidad y discapacidad a nivel mundial. Los registros de la mortalidad que se asocian con el consumo de alcohol están fraccionados. El objetivo de este estudio fue escribir la mortalidad relacionada con la ingesta de alcohol en pacientes con cirrosis atendidos en un hospital universitario de la ciudad de Medellín. Materiales y métodos: se incluyeron 163 pacientes con diagnóstico de cirrosis, evaluados en la consulta externa de hepatología de un hospital de referencia en la ciudad de Medellín con 277 camas y seguimiento hasta el 2016. Se midieron variables sociodemográficas, paraclínicas y clínicas. Se consideró el consumo de alcohol al inicio del seguimiento. Se describió la supervivencia y las complicaciones asociadas con la cirrosis según el estado de consumidores vs. no consumidores de alcohol. Resultados: se siguieron 163 pacientes hasta diciembre del 2016, encontrando una mortalidad en el $51 \%$ en consumidores de alcohol vs. $39 \%$ en no consumidores $(P=0,19)$. Las complicaciones de la cirrosis en consumidores de alcohol fueron ascitis en $68 \%$ vs. $43 \%(P=0,01)$ en el grupo sin consumo de alcohol, encefalopatía $40,6 \%$ vs. 13,5\% ( $P=0,00)$ y carcinoma hepatocelular (HCC) en $29 \%$ vs. $17 \%(P=0,08)$. En el análisis por subgrupos, los pacientes con hepatitis $C$ con consumo de alcohol tuvieron una mortalidad más alta comparado con los pacientes que no consumieron alcohol (OR 33, IC 95\%: 1,06 a 1023). Conclusiones: a pesar que el consumo de alcohol no se relaciona con aumento de la mortalidad en pacientes con cirrosis en este estudio, sí se observa incremento de esta en ciertas poblaciones, como en el subgrupo de pacientes con hepatitis C.
\end{abstract}

Palabras clave

Cirrosis, consumo de alcohol, alcoholismo, mortalidad, hepatopatía crónica.

\begin{abstract}
Worldwide, alcohol consumption is a well-known risk factor for premature death, morbidity and disability. Records of mortality associated with alcohol consumption are not centralized. The aim of this study was to record the mortality rate associated with alcohol intake in patients with cirrhosis who were treated at a university hospital in the city of Medellin. Materials and methods: We included 163 patients who had been diagnosed with cirrhosis in the outpatient hepatology clinic of a 277 bed referral hospital in Medellín. Patients were monitored until 2016. Sociodemographic, paraclinical and clinical variables were measured. Alcohol consumption was considered at the beginning of the follow-up. Survival and complications associated with cirrhosis were described and recorded for patients who consumed alcohol as well as for those who did not, and then the two groups were compared. Results: One hundred sixty-three patients were followed until December 2016. The mortality rate among those who consumed alcohol was $51 \%$ while it was only $39 \%$ for those who did not consume alcohol $(P=0.19)$. Comparison of complications of cirrhosis showed that $68 \%$ of alcohol users developed ascites vs. $43 \%$ of non-consumers $(P=0.01) ; 40.6 \%$ of alcohol users developed encephalopathy vs. $13.5 \%$ of non-consumers $(P=0.00)$; and $29 \%$ of alcohol users developed hepatocellular carcinoma (HCC) vs. $17 \%$ of non-consumers $(P=0.08)$. In the subgroup analysis, patients with hepatitis $C$ who consumed alcohol had a higher mortality rate than patients who did not consume alcohol (OR: 33, 95\% Cl: 1.06 to 1023). Conclusions: Although alcohol consumption was not related to increased mortality among patients with cirrhosis in this study, increased mortality was observed in the subgroup of patients with hepatitis $C$.
\end{abstract}

Keywords

Cirrhosis, alcohol consumption, alcoholism, mortality, chronic liver disease. 


\section{INTRODUCCIÓN}

El consumo de alcohol es un conocido factor de riesgo para muerte prematura, morbilidad y discapacidad a nivel mundial. En Colombia, cerca de 7 millones de personas entre los 12 y 65 años consumen alcohol, y $35 \%$ tiene una ingesta superior de 100 g/día (1). En Francia, 33\% de los casos de fibrosis hepática se relaciona con el consumo pesado de alcohol, y este es responsable de 3,8\% de la mortalidad global, especialmente en jóvenes $(2,3)$. La situación no es muy diferente en Estado Unidos, donde se considera la segunda causa de trasplante hepático, y $44 \%$ de las muertes por enfermedad hepática avanzada está asociada con el consumo de alcohol $(4,5)$.

El espectro de la enfermedad hepática por alcohol es amplio y comprende diferentes estadios, que pueden ser consecutivos o estar superpuestos, haciendo su estudio complejo (6). La esteatohepatitis se describe en cerca de $90 \%$ de los consumidores de más de $80 \mathrm{~g} /$ día de alcohol (7). Tras un consumo alto y continuo de alcohol puede desarrollarse hepatitis alcohólica, donde la mortalidad alcanza el $35 \%$, y si esta se presenta en individuos con cirrosis, el pronóstico es aún más ominoso $(8,9)$.

En el estudio Diosysos, donde se siguió una cohorte de 6543 pacientes, se describió la prevalencia de enfermedad hepática inducida por alcohol en una comunidad italiana, relacionándose el consumo de más de $30 \mathrm{~g} /$ día con un aumento del riesgo para el desarrollo de cirrosis hepática, y el consumo de más de $120 \mathrm{~g}$ / día de alcohol incrementó 63 veces el riesgo de desarrollar cirrosis. Aun así, la prevalencia de cirrosis fue apenas de 13,5\% (10).

Se han estudiado también los patrones de consumo de alcohol asociados con desarrollo de cirrosis y enfermedad hepática, especialmente en los consumidores ocasionales de grandes cantidades de alcohol. Los atracones, entendidos como una ingesta de alcohol suficiente para elevar la concentración de alcohol en sangre por encima de 0,08 g/ $\mathrm{dL}$ en 2 horas, se han considerado factores de riesgo para cirrosis (11). Además de la cantidad y patrón de consumo, el género también se ha relacionado con un mayor riesgo de cirrosis; por ejemplo, las mujeres tienen mayor predisposición de sufrir los efectos a largo plazo, necesitando menores cantidades de alcohol que los hombres para terminar desarrollando cirrosis hepática (12-15).

El alcohol tiene una relación directa con el desarrollo de enfermedad hepática terminal; además, cuando coexiste con otras enfermedades, como las hepatitis virales, su efecto nocivo se ve potenciado, generando peores desenlaces, y su consumo se asocia también con mayores tasas de infección y cáncer $(13,16-21)$. En Colombia, la información acerca de la ingesta de alcohol en el contexto de la enfermedad hepática está fragmentada, y solo contamos con algunos reportes aislados (22). Esta se refiere como la primera causa de cirrosis hepática en una población de pacientes en Pereira, donde se caracterizaron 100 pacientes con cirrosis, y se describió el consumo de alcohol en $23 \%$ de los casos. Esto contrasta con el reporte de 2 centros de referencia en la ciudad de Bogotá, donde se siguió una cohorte de 419 pacientes con cirrosis hepática, y se refirió el consumo de alcohol como la segunda causa de cirrosis en $14 \%$ de los pacientes $(23,24)$. En la ciudad de Medellín, un reporte de experiencia de trasplante hepático en el Hospital Pablo Tobón Uribe mostró la cirrosis por alcohol como la segunda causa de trasplante hepático entre en 2004 y 2010 (25). También, el consumo de alcohol fue factor de riesgo para carcinoma hepatocelular en un estudio realizado entre el 2005 y el 2007 en la misma institución (26). El objetivo de este estudio fue describir el efecto del consumo de alcohol en la sobrevida de pacientes con cirrosis hepática en una cohorte de pacientes de la ciudad de Medellín.

\section{METODOLOGíA}

Se realizó un estudio observacional descriptivo de cohorte retrospectiva de pacientes atendidos en la consulta externa de hepatología del Hospital Pablo Tobón Uribe, institución de cuarto nivel de atención en la ciudad de Medellín, con 277 camas, durante el 2004 al 2006 con los siguientes criterios de inclusión: mayores de 18 años; diagnóstico de cirrosis hepática mediante imagen o biopsia hepática; seguimiento hasta diciembre del 2016.

Se evaluaron variables sociodemográficas, bioquímicas y clínicas al inicio del seguimiento. Se estratificó a los pacientes según su consumo de alcohol en el año previo al diagnóstico como: alto, mayor de $80 \mathrm{~g} /$ día en hombres o más de $40 \mathrm{~g} /$ día en mujeres; moderado, entre 50 y $80 \mathrm{~g}$ /día en hombres o entre 20 y $40 \mathrm{~g} /$ día en mujeres; bajo, cuando el consumo de alcohol fue menor de $50 \mathrm{~g} /$ día en hombres o de $20 \mathrm{~g} /$ día en mujeres; y los no consumidores. Para el cálculo de mortalidad y complicaciones asociadas con el consumo de alcohol se estratificó a los pacientes entre consumidores, sin considerar la cantidad, y no consumidores de alcohol.

Las variables categóricas se presentan como frecuencias absolutas y relativas, y las variables continuas como mediana, rango y rango intercuartílico. Para valorar la supervivencia de los pacientes se utilizó el método de Kaplan-Meier. Para el análisis bivariado se usó Logrank, con un valor de significancia estadística de $p<0,05$. El desenlace de interés para este análisis fue muerte, y los pacientes trasplantados se censuraron. La tabulación y análisis de los datos se hicieron mediante el programa Epidat versión 4.2, y se realizaron curvas de supervivencia con SPSS versión 21. El estudio fue aprobado por el Comité de Ética del Hospital Pablo Tobón Uribe, y según Resolución 8430 de 1993 se clasificó como un estudio 
sin riesgo. Los investigadores se adhieren a la Declaración de Helsinki versión 2013.

\section{RESULTADOS}

Se incluyeron 163 pacientes adultos con diagnóstico de cirrosis hepática, 64\% fue de sexo masculino (Tabla 1). La mediana de la edad fue de 60 años, rango intercuartil (RIC) entre 52 y 66 años, $68 \%$ era de raza mestiza, según reporte de la historia clínica. La causa más frecuente de cirrosis fue el consumo de alcohol en 39\% (64/163) de los pacientes. $35,5 \%$ de los pacientes se clasificó como Child Turcotte Pugh (CTP) A al inicio del seguimiento. Las complicaciones observadas relacionadas con la cirrosis fueron ascitis en $57 \%$ de los pacientes seguida de coagulopatía en 36\%, sangrado variceal en $32 \%$, encefalopatía en $28 \%$ y carcinoma hepatocelular en $23 \%$.

Con respecto al consumo de alcohol, $45,4 \%$ no reportó consumo del mismo; $44,2 \%$ de los pacientes informó consumo de alcohol alto; $6,1 \%$ consumo bajo y $4,2 \%$ consumo moderado. Al categorizar a los pacientes según el consumo de alcohol, $89 \%$ de los consumidores de alcohol fue hombre, con una mediana de 60 años (RIC entre 29 y 82 años); mientras en el grupo que no tuvo consumo de alcohol, $67 \%$ fue mujer $(P=0,00)$. Como complicaciones asociadas con la cirrosis, en el grupo con consumo de alcohol se encontró ascitis como principal complicación en $68 \%$ vs. $43 \%(P=$ $0,00)$ en el grupo sin consumo de alcohol. Se clasificaron como CTP B 37 pacientes (41\%), CTP A 26 pacientes (29\%) y CTP C 26 pacientes (29\%) (Tabla 2).

La mortalidad fue del $45 \%$ (74/163), con una mediana de sobrevida de 4 años y 2 meses después del diagnóstico. 53 pacientes (33\%) se llevaron a trasplante hepático tras una mediana de 3 años y 4 meses. De los pacientes consumidores de alcohol, 45 pacientes $(51 \%)$ murieron tras una mediana de 3 años y 10 meses comparado con 4 años y 2 meses en el grupo de no consumidores de alcohol $(P=$ 0,9). El OR para muerte fue de 1,63 (IC 95\%: 0,87 a 3,05). Fueron CPT B 21 pacientes (46\%), CPT C 13 pacientes (28\%) y CTP A 11 pacientes (25\%).

En el análisis por subgrupos según la etiología de la cirrosis tuvieron cirrosis de etiología autoinmune 17 pacientes (10,4\%), todos ellos sin consumo de alcohol; de estos, 10 pacientes como CTP B (59\%) y 7 pacientes clasificados como CTP A (41\%). Tampoco tuvieron consumo de alcohol 10 pacientes con otras etiologías, 1 de ellos con hemocromatosis hereditaria clasificado como CTP C y los 10 pacientes restantes con cirrosis de etiología biliar, 1 paciente catalogado como CTP 3 (5\%), 4 como CTP 2.

19 pacientes $(11,7 \%)$ tuvieron cirrosis por hepatitis B, 9 de ellos (47\%) reportaron consumo de alcohol, 3 con un consumo alto. Murieron 6 pacientes $(6 / 19,31 \%), 2$ pacientes
Tabla 1. Características demográficas y clínicas en 163 pacientes con diagnóstico de cirrosis hepática atendidos en el Hospital Pablo Tobón Uribe entre 2004 a 2006.

\begin{tabular}{lc}
\hline & $\mathrm{n}=163$ \\
\hline Edad (mediana, RIC) & 60 años (52-66) \\
Sexo & \\
Masculino & $105(64,4 \%)$ \\
Femenino & $58(35,6 \%)$ \\
Raza ${ }^{1}$ & \\
Mestizo & $111(68 \%)$ \\
Negro & $3(1,8 \%)$ \\
Blanco & $49(30,1 \%)$ \\
Indígena & 0 \\
Clasificación de Child & \\
A & \\
B & $58(35,5 \%)$ \\
C & $65(39,9 \%)$ \\
Etiología de la cirrosis & $40(24,5 \%)$ \\
Alcohol & \\
Criptogénica & $64(39,6 \%)$ \\
Autoinmune & $31(19 \%)$ \\
Hepatitis B & $17(10,4 \%)$ \\
NASH & $15(9,2 \%)$ \\
Hepatitis C & $12(7,3 \%)$ \\
Biliar & $9(5,4 \%)$ \\
Alcohol y virus B & $8(5 \%)$ \\
Hemocromatosis & $4(2,5 \%)$ \\
Alcohol y virus C & $1(0,6 \%)$ \\
Moderado ${ }^{4}$ & \\
\hline
\end{tabular}

${ }^{1}$ Raza según reporte en la historia clínica.

${ }^{2}$ Más de $80 \mathrm{~g} /$ día de alcohol en hombres, y más de $40 \mathrm{~g} /$ día en mujeres. ${ }^{3}$ Entre 50 y $80 \mathrm{~g} /$ día de alcohol en hombres, y entre 20 y $40 \mathrm{~g} /$ día en mujeres.

${ }^{4}$ Menos de $50 \mathrm{~g} /$ día de alcohol en hombres, y menos de $20 \mathrm{~g} /$ día de alcohol en mujeres.

$(2 / 9,22 \%)$ con consumo de alcohol y 4 pacientes $(4 / 10$, 40\%) sin consumo de alcohol (OR 0,43; IC 95\%: 0,060 a 3,22; $P=0,4) .9$ pacientes $(47 \%)$ fueron llevados a trasplante hepático, 5 pacientes (55\%) consumidores de alcohol y $4(40 \%)$ sin consumo de alcohol (OR 1,88; IC 95\%: 0,3 a 11,$63 ; P=0,4) .3$ pacientes $(33 \%)$ tuvieron $\mathrm{HCC}$ en el grupo de consumidores de alcohol y 3 pacientes $(30 \%)$ tam- 
Tabla 2. Comparación de desenlaces entre consumidores y no consumidores de alcohol en una cohorte de 163 pacientes con diagnóstico de cirrosis hepática atendidos en el Hospital Pablo Tobón Uribe.

\begin{tabular}{|c|c|c|c|c|}
\hline & $\begin{array}{c}\text { Consumo de alcohol }{ }^{1} \\
n=89\end{array}$ & $\begin{array}{c}\text { No consumo de alcohol }{ }^{2} \\
n=74\end{array}$ & & \\
\hline Supervivencia & & & OR (IC 95\%) & $\mathrm{P}$ \\
\hline Muerte & $45(51 \%)$ & $29(39 \%)$ & $1,63(0,87$ a 3,05$)$ & 0,129 \\
\hline Trasplante & $27(30 \%)$ & $26(36 \%)$ & $0,89(0,4$ a 1,7$)$ & 0,26 \\
\hline Tiempo entre el diagnóstico y la muerte & $\begin{array}{c}3 \text { años } 10 \text { meses } \\
\text { (5 meses- } 7 \text { años } 10 \text { meses) }\end{array}$ & $\begin{array}{c}4 \text { años } 2 \text { meses } \\
\text { (18 meses-6 años } 5 \text { meses) }\end{array}$ & & 0,9 \\
\hline Tiempo entre el diagnóstico y el trasplante & $\begin{array}{c}3 \text { años } 3 \text { meses } \\
\text { (23 meses- } 4 \text { años } 10 \text { meses) }\end{array}$ & $\begin{array}{c}3 \text { años } 4 \text { meses } \\
\text { (1 año-5 años } 6 \text { meses) }\end{array}$ & & 0,7 \\
\hline \multicolumn{5}{|l|}{ Complicaciones de la cirrosis } \\
\hline Encefalopatía & $37(41 \%)$ & $10(13 \%)$ & $\begin{array}{c}4,55 \\
2,1 \text { a } 10\end{array}$ & 0,001 \\
\hline Ascitis & $60(68 \%)$ & $32(43 \%)$ & $\begin{array}{c}2.72 \\
1,4 \text { a } 5,1\end{array}$ & 0,001 \\
\hline Peritonitis bacteriana espontánea & $4(4 \%)$ & $4(5 \%)$ & $\begin{array}{c}0,8 \\
0,2 \text { a } 3,4\end{array}$ & 0,8 \\
\hline Sangrado variceal & $32(36 \%)$ & $19(25 \%)$ & $\begin{array}{c}1,6 \\
0,8 \text { a } 3,2\end{array}$ & 0,11 \\
\hline Coagulopatía & $39(44 \%)$ & $19(25 \%)$ & $\begin{array}{c}2,26 \\
1,2 \mathrm{a} 4,4\end{array}$ & 0,01 \\
\hline Síndrome hepatorrenal & $4(4 \%)$ & $0 \%$ & & \\
\hline $\mathrm{HCC}$ & $26(29 \%)$ & $13(17 \%)$ & $\begin{array}{c}1,94 \\
0,91 \text { a } 4,1\end{array}$ & 0,08 \\
\hline Hepatitis alcohólica & $14(16 \%)$ & $0 \%$ & & \\
\hline \multicolumn{5}{|l|}{ Paraclínicos } \\
\hline Bilirrubina & 2,9 & 2,4 & & 0,2 \\
\hline INR & 1,3 & 1,2 & & 0,05 \\
\hline Albúmina & 3,2 & 3,3 & & 0,6 \\
\hline Creatinina & 1 & 0,9 & & 0,3 \\
\hline
\end{tabular}

${ }^{1}$ Cualquier consumo de alcohol alto, moderado o bajo.

${ }^{2}$ Sin consumo de alcohol.

bién presentaron HCC en el grupo de no consumidores de alcohol (OR 1,17; IC 95\%: 0,17 a 8,09; $P=0,8$ ).

De los 10 pacientes con cirrosis por hepatitis C, $5(50 \%)$ reportaron consumo de alcohol, y 5 (50\%) fueron abstemios, de estos 2 CTP B, 2 CTP C y 1 CTP A. Murieron 4 pacientes durante el seguimiento, todos ellos consumidores de alcohol (OR 33; IC 95\%: 1,06 a 1023), de los cuales 2 de ellos fue CTP B, 1 CTP A y 1 CTP C. Fueron llevados a trasplante hepático 5 pacientes, 4 de ellos (80\%) sin consumo de alcohol y 1 paciente $(20 \%)$ consumidor de alcohol, CTP A con HCC (OR 2,25; IC 95\%: 0,06 a 87; $P$ $=0,058$ ). Hubo 2 casos de HCC, 1 en el grupo de consumidores de alcohol y otro en el grupo de no consumidores.

De los 31 pacientes con cirrosis de etiología criptogénica hubo 8 (25\%) con consumo de alcohol en cantidad moderada, y $23(74 \%)$ sin consumo de alcohol. Murieron 18 pacientes (58\%), 6 (75\%) consumidores de alcohol y 12 (52\%) no consumidores de alcohol (OR 2,75; IC 0,46 a 16; $P=0,2)$. Se llevaron a trasplante hepático 9 pacientes (29\%), 2 del grupo de consumidores de alcohol (25\%) y 7 (30\%) del grupo sin consumo de alcohol (OR 0,76; IC 95\%: 0,1 a 4,7; $P=0,4) .10$ pacientes tuvieron HCC, 4 (50\%) con consumo de alcohol y 6 (26\%) sin consumo de alcohol (OR 1,83; IC 0,53 a 15) (Figura 1).

12 pacientes $(7,3 \%)$ tuvieron cirrosis por esteatohepatitis no alcohólica, 3 de ellos reportaron consumo de alcohol en baja cantidad (25\%); 8 pacientes (66\%) murieron, 2 de los consumidores de alcohol (66\%) y 6 abstemios (66\%); 1 paciente de cada grupo fue llevado a trasplante hepático (OR 4; IC 95\%: 0,17 a 95; $P=0,3$ ). 
De los 64 pacientes que tuvieron cirrosis por alcohol, 62 (96\%) presentaron consumo alto y 2 (4\%) consumo moderado. Murieron 31 pacientes en el seguimiento (41\%), 30 de estos con un consumo alto de alcohol (OR 0,94; IC 0,06 a 15). De los 17 pacientes llevados a trasplante hepático, 16 tuvieron consumo alto de alcohol (OR 0,35; IC 95\%: 0,02 a 5,8); y 15 de los 16 casos de HCC también mostraron consumo alto de alcohol.

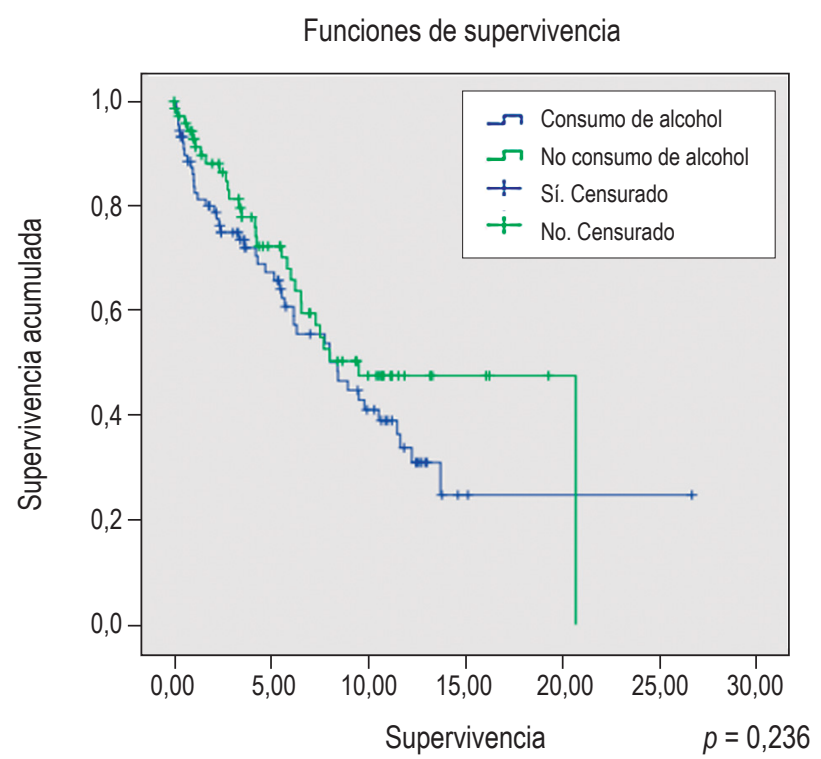

Figura 1. Supervivencia de los pacientes según el consumo de alcohol.

\section{DISCUSIÓN}

El consumo de alcohol ha sido un tema de interés en la población colombiana, la cual cuenta con una estrategia nacional frente al consumo de alcohol en Colombia (1). Se han caracterizado los pacientes con cirrosis en la ciudad de Pereira y Bogotá en dos centros de referencia, y en la ciudad de Medellín en el Hospital Pablo Tobón Uribe, donde se describieron las características de los pacientes llevados a trasplante hepático. Con este estudio se buscó describir las características de una cohorte de 163 pacientes con diagnóstico de cirrosis hepática seguida por 10 años en donde también se cuantificó el consumo de alcohol para evaluar el efecto que podría tener este en la sobrevida de los pacientes con cirrosis establecida (24).

El consumo de alcohol fue la causa más frecuente de cirrosis en esta cohorte, situación similar a la observada en la cohorte reportada en la ciudad de Pereira y las cohortes seguidas en Europa y Estados Unidos (3, 23, 28). Con respecto a las características demográficas de los pacientes se observaron pacientes en la séptima década de la vida, predominantemente hombres, con severidad de la enfer- medad hepática variable. Al inicio del seguimiento $2 / 3$ de los pacientes tuvo cirrosis descompensada, siendo CTP B y C el 65\% de la población descrita. Esto probablemente en relación con el acceso tardío de los pacientes a instituciones con alto nivel de complejidad, como es el Hospital Pablo Tobón Uribe, al momento del desarrollo de complicaciones asociadas con la cirrosis.

La hepatitis B y C se observó en 17\% de los pacientes. Con respecto al consumo de alcohol, cerca del 50\% de la población reportó algún tipo de consumo, la mayoría fue alto y muy pocos pacientes, apenas el $6 \%$ con consumo bajo, este último tipo de consumo se ha encontrado en poblaciones europeas y orientales donde se ha llegado a relacionar con disminución en la mortalidad de origen cardiovascular en pacientes no cirróticos, y reducción en la presencia de esteatohepatitis en pacientes japoneses $(28$, 29). Sin embargo, en nuestra población muy pocos pacientes tuvieron un consumo bajo de alcohol.

La mortalidad de los consumidores de alcohol no fue diferente a la del grupo de no consumidores, y el tiempo de vida entre el diagnóstico de cirrosis y la muerte fue mayor en el grupo de no consumidores de alcohol, sin ser esto estadísticamente significativo; esto explicado por el acceso de los pacientes al trasplante hepático. $27 \%$ de los pacientes consumidores de alcohol fue llevado a trasplante hepático, situación que altera la historia natural del consumo de alcohol. De los 53 pacientes trasladados a trasplante, 16 (30\%) tenían cirrosis por alcohol, siendo la principal causa de trasplante en el hospital Pablo Tobón Uribe; ninguno de estos pacientes con hepatitis alcohólica como indicación de trasplante.

La relación entre consumo de alcohol y mortalidad por cirrosis se ha descrito en múltiples cohortes, observándose que la cantidad, patrón de consumo, así como el tipo de bebidas son determinantes en el desarrollo de enfermedad hepática y muerte relacionada con cirrosis. Esto constituye una limitante para los estudios ya que, durante períodos de seguimiento largos, como el reportado en este estudio, los patrones de consumo, así como la cantidad y características de las bebidas, tienden a variar con el tiempo, haciendo la evaluación de esos determinantes imposible. Cuando se detalla el consumo de alcohol asociado con las diferentes etiologías de cirrosis hepática, observamos un aumento en la mortalidad en los pacientes con hepatitis $\mathrm{C}$, donde todos los pacientes fallecidos tuvieron un consumo de alcohol alto, y parecían estar tan enfermos como los no consumidores con CTP parecidos, situación similar a la reportada en la literatura. Los pacientes con cirrosis hepática por autoinmunidad no reportaron consumo de alcohol, así como aquellos con otras etiologías de cirrosis.

Con respecto a las complicaciones asociadas con cirrosis, evidentemente los pacientes con consumo de alcohol tuvieron más complicaciones que aquellos sin consumo de alcohol, 
situación que podría traducirse en mayor tiempo de estancia hospitalaria, con sus respectivos costos, el cual necesitaría estudios adicionales, y que no se consideró en este estudio.

Dentro de las fortalezas del estudio está el seguimiento a 10 años de todos los pacientes, así como el tamaño de la cohorte. La cuantificación del consumo de alcohol permitió establecer la relación entre cantidad de alcohol consumida y aumento en la mortalidad en algunos escenarios, como el caso de la hepatitis C.

Haber categorizado el consumo de alcohol según lo reportado en la literatura internacional podría verse como limitación, ya que la población colombiana, específicamente de la ciudad de Medellín, podría necesitar cantidades diferentes de alcohol para llegar a morir por cirrosis. El estudio se planteó para determinar la mortalidad por cualquier causa de los pacientes incluidos, lo que constituye también una limitación. Bajo este planteamiento tampoco se evaluaron otras variables, como hospitalizaciones durante el seguimiento. Además, cabe señalar que el $30 \%$ de los pacientes con consumo de alcohol fue llevado a trasplante hepático.

\section{CONCLUSIONES}

Aunque el consumo de alcohol no aumentó la mortalidad en esta cohorte, sí disminuyó la sobrevida de los pacientes con hepatitis C con clasificaciones de CTP similares. El consumo de alcohol fue la principal causa de cirrosis en esta cohorte, y el consumo aumentó la presencia de complicaciones de cirrosis, que adicionalmente son signos de descompensación de la enfermedad hepática. Con respecto al patrón de consumo de alcohol, esta cohorte tiene consumos extremos, siendo la distribución muy similar entre consumo alto y no consumo de alcohol. El trasplante hepático en esta población cambia la historia natural de enfermedad, situación que podría explicar la mortalidad similar en los dos grupos en nuestra cohorte con respecto a lo reportado en las otras cohortes referenciadas.

\section{Agradecimientos}

Luis Enrique Martínez R, Luisa Calle, Juan David Vélez, Natalia Giraldo, Juan Camilo Pérez.

\section{Financiamiento}

Departamento Administrativo de Ciencia, Tecnología e Innovación, Colciencias (111556935008).

\section{REFERENCIAS}

1. Ministerio de Salud. Estrategia nacional de respuesta integral frente al consumo de alcohol en Colombia. 2013.
Disponible en: https://www.minsalud.gov.co/sites/rid/ Lists/BibliotecaDigital/RIDE/VS/PP/ENT/estrategianacional-alcohol-colombia.pdf

2. Roulot D, Costes JL, Buyck JF, Warzocha U, Gambier N, Czernichow S, et al. Transient elastography as a screening tool for liver fibrosis and cirrhosis in a community-based population aged over 45 years. Gut. 2010;60:977-84. doi: https://doi.org/10.1136/gut.2010.221382.

3. European Association for the Study of the Liver. EASL clinical practical guidelines: management of alcoholic liver disease. J Hepatol. 2012;57(2):399-420. doi: https://doi. org/10.1016/j.jhep.2012.04.004.

4. Sherlock S. Alcoholic liver disease. Lancet. 1995; 345 (8944): 227-9. doi: https://doi.org/10.1016/S0140-6736 (95)90226-0.

5. Yoon YH, Yi HY, Thomson PC. Liver cirrhosis mortality in the United States, 1970- 2009, surveillance report \#93. National Institute on Alcohol Abuse and Alcoholism. 2012.

6. Plunk AD, Syed-Mohammed H, Cavazos-Rehg P, Bierut LJ, Grucza RA. Alcohol consumption, heavy drinking, and mortality: rethinking the j-shaped curve. Alcohol Clin Exp Res. 2014;38(2):471-8. doi: https://doi.org/10.1111/acer.12250.

7. Dugum M, Mccullough A. Diagnosis and management of alcoholic liver disease. J Clin Transl Hepatol. 2015;3(2):10916. doi: 10.14218/JCTH.2015.00008.

8. Mathurin P, O'Grady J, Carithers RL, Phillips M, Louvet A, Mendenhall CL, et al. Corticosteroids improve short-term survival in patients with severe alcoholic hepatitis: metaanalysis of individual patient data. Gut. 2011;60(2):255-60. doi: https://doi.org/10.1136/gut.2010.224097.

9. Mathurin P, Moreno C, Samuel D, Dumortier J, Salleron J, Durand F, et al. Early liver transplantation for severe alcoholic hepatitis. N Engl J Med. 2011;365(19):1790-800. doi: 10.1056/NEJMoa1 105703.

10. Bellentani S, Saccoccio G, Costa G, Tiribelli C, Manenti F, Sodde M, et al. Drinking habits as cofactors of risk for alcohol induced liver damage. The Dionysos Study Group. Gut. 1997;41:845-50. doi: https://doi.org/10.1136/ gut.41.6.845.

11. Chacko KR, Reinus J. Spectrum of alcoholic liver disease. Clin Liver Dis. 2016;20(3):419-27. doi: https://doi. org/10.1016/j.cld.2016.02.002.

12. SaitzR. Unhealthyalcoholuse. NEnglJMed.2005;352(6):596607. doi: https://doi.org/10.1056/NEJMcp042262.

13. Chan C, Levitsky J. Infection and alcoholic liver disease. Clin Liver Dis. 2016;20(3):595-606. doi: https://doi. org/10.1016/j.cld.2016.02.014.

14. Mandayam S, Jamal MM, Morgan TR. Epidemiology of alcoholic liver disease. Semin Liver Dis. 2004;24:217-32. doi: https://doi.org/10.1055/s-2004-832936.

15. Lieber CS, Jones DP, Decarli LM. Effects of prolonged ethanol intake: production of fatty liver despite adequate diets. J Clin Invest. 1965;44:1009-21. doi: https://doi. org/10.1172/JCI105200.

16. Albanis E, Friedman SL. Pathogenesis and principles of therapy. Curr Futur Treat Ther Liver Dis. 2001;5(2):315-34. 
17. Larkin J, Clayton MM, Liu J, Feitelson MA. Chronic ethanol consumption stimulates hepatitis B virus gene expression and replication in transgenic mice. Hepatology. 2001;34:792-7. doi: https://doi.org/10.1053/jhep.2001.27565.

18. Westin J, Lagging LM, Spak F, Aires N, Svensson E, Lindh $\mathrm{M}$, et al. Moderate alcohol intake increases fibrosis progression in untreated patients with hepatitis $\mathrm{C}$ virus infection. $\mathrm{J}$ Viral Hepat. 2002;9:235-41. doi: https://doi.org/10.1046/ j.1365-2893.2002.00356.x.

19. Lin CW, Lin CC, Mo LR, Chang CY, Perng DS, Hsu CC, et al. Heavy alcohol consumption increases the incidence of hepatocellular carcinoma in hepatitis B virus-related cirrhosis. J Hepatol. 2013;58:730-5. doi: https://doi. org/10.1016/j.jhep.2012.11.045.

20. Seitz H, Sticke LF. Molecular mechanisms of alcohol-mediated carcinogenesis. Nat Rev Cancer. 2007;7(8):599. doi: https://doi.org/10.1038/nrc2191.

21. Jee SH, Ohrr H, Sull JW, Samet JM. Cigarette smoking, alcohol drinking, hepatitis B, and risk for hepatocellular carcinoma in Korea. J Natl Cancer Inst. 2004;96:1851-6. doi: https://doi.org/10.1093/jnci/djh334.

22. Suárez Quintero Y. Consumo de alcohol en Colombia: ¿sabemos en qué estamos? Rev Colomb Gastroenterol. 2015;30(4):387-8.

23. Giraldo A, Barraza M, Villa H, Martínez J, García G. Caracterización epidemiológica de pacientes con cirrosis en una consulta de gastroenterología en Pereira, Colombia, 2009-2012. Rev Med Risaralda. 2014;20(5):86-94.
24. Prieto JE, Sánchez S, Prieto RG, Rojas EV, González L, Mendivelso F. Características clínicas y descompensación en pacientes con cirrosis hepática atendidos en dos centros de hepatología en la ciudad de Bogotá DC, 2010-2014. Rev Colomb Gastroenterol. 2016;31(1). doi: http://dx.doi. org/10.22516/25007440.66.

25. Santos O, Londoño M, Marín J, Muñoz O, Mena A, Guzmán $\mathrm{C}$, et al. An experience of liver transplantation in Latin America: a medical center in Colombia. Colomb Med. 2015;46:8-13.

26. Cortes-Mancera F, Loureiro CL, Hoyos S, Restrepo JC, Correa G, Jaramillo S, et al. Etiology and viral genotype in patients with end-stage liver diseases admitted to a hepatology unit in Colombia. Hepat Res Treat. 2011;2011:363205. doi: $10.1155 / 2011 / 363205$.

27. Singal AK, Kamath PS, Gores GJ, Shah VH. Alcoholic hepatitis: current challenges and future directions. Clin Gastroenterol Hepatol. 2014;12(4):555-64. doi: 10.1016/j. cgh.2013.06.013.

28. Chiva-Blanch G, Arranz S, Lamuela-Raventos RM, Estruch R. Effects of wine, alcohol and polyphenols on cardiovascular disease risk factors: evidences from human studies. Alcohol Alcohol. 2013;48(3):270-7. doi: 10.1093/alcalc/agt007.

29. Sogabe M, Okahisa T, Taniguchi T, Tomonari T, Tanaka T, Tanaka $\mathrm{H}$, et al. Light alcohol consumption plays a protective role against non - Alcoholic fatty liver disease in Japanese men with metabolic syndrome. Liver Int. 2015;35(6)170714. doi: 10.1111/liv.12754. 